\title{
Optoelectronic properties of graphene in the presence of optical phonon scattering
}

\author{
W. Xu, ${ }^{1,2, *}$ H. M. Dong, ${ }^{1}$ L. L. Li, ${ }^{1}$ J. Q. Yao, ${ }^{3}$ P. Vasilopoulos,${ }^{4}$ and F. M. Peeters ${ }^{5}$ \\ ${ }^{1}$ Key Laboratory of Materials Physics, Institute of Solid State Physics, Chinese Academy of Sciences, Hefei 230031, China \\ ${ }^{2}$ Department of Physics, Yunnan University, Kunming 650091, China \\ ${ }^{3}$ Institute of Laser \& Opto-electronics, Tianjin University, Tianjin 300072, China \\ ${ }^{4}$ Department of Physics, Concordia University, Montréal, Québec, Canada H3G 1M8 \\ ${ }^{5}$ Department of Physics, University of Antwerp, Groenenborgerlaan 171, B-2020 Antwerpen, Belgium \\ (Received 28 May 2010; revised manuscript received 15 August 2010; published 3 September 2010)
}

\begin{abstract}
We study in detail the optoelectronic properties of graphene. Considering the electron interactions with photons and phonons, we employ the mass- and energy-balance equations to self-consistently evaluate the photoinduced carrier densities, the optical conductance, and the transmission coefficient in the presence of a linearly polarized radiation field. We demonstrate that the photoinduced carrier densities increase around the electron-photon-phonon resonant transition. They depend strongly on the radiation intensity and frequency, temperature, and dark carrier density. For short-wavelength radiation $(\mathcal{L}<3 \mu \mathrm{m})$, we obtain the universal optical conductance $\sigma_{0}=e^{2} /(4 \hbar)$. Importantly, there exists an optical-absorption window in the radiation wavelength range 4-100 $\mu \mathrm{m}$, which is induced by different transition energies required for interband and intraband optical absorption. The position and width of this window depend sensitively on the temperature and the carrier density of the system. These theoretical results are in line with recent experimental findings and indicate that graphene exhibits important features not only in the visible regime but also in the midinfrared bandwidth.
\end{abstract}

DOI: 10.1103/PhysRevB.82.125304

PACS number(s): 78.20.Bh, 78.20.Ci

\section{INTRODUCTION}

Since the experimental discovery of graphene in $2004,{ }^{1}$ the investigation of graphene-based electronics and optoelectronics has quickly become one of the most important research topics in condensed-matter physics, nanomaterial science, and nanoelectronics. ${ }^{2,3}$ Due to its excellent electronic transport, optical, and optoelectronic properties, such as high carrier density and mobility along with the high optical transmittance in the air-graphene-wafer systems, graphene has been proposed as an advanced material for new generation of electronic and optoelectronic devices. Graphene-based electronic devices exhibit high carrier mobility and quasiballistic transport over submicron scales even at room temperature. ${ }^{4}$ It has already been used to realize high-speed electronic devices such as field-effect transistors, ${ }^{5} p$ - $n$ junctions, ${ }^{6}$ highfrequency devices, ${ }^{7}$ to mention but a few. Very recently, graphene has also been proposed as an advanced transparent conducting material by utilizing its combined excellent transport and optical properties. ${ }^{8}$ It has been shown that graphene can be used to replace conventional indium tin oxide transparent electrodes for making better and cheaper optical displays. ${ }^{9}$ Presently, graphene-based transparent electronics is a hot field of research for both fundamental studies and device applications. ${ }^{8}$

For the use of graphene as an optoelectronic and transparent electronic device, the investigation of its optical and optoelectronic properties is critical and essential. Recent experimental and theoretical work has demonstrated and predicted some particular and interesting optoelectronic properties in the infrared-to-visible spectral range for air-graphenewafer systems. In particular, the results obtained from optical transmission $^{10}$ and infrared absorption ${ }^{11}$ measurements show the following features. (i) The optical conductance per graphene layer is a universal value $\sigma_{0}=e^{2} /(4 \hbar)$ in the visible frequency range, ${ }^{10,11}$ which can be viewed as an intrinsic property of two-dimensional (2D) massless fermions. (ii) The corresponding light transmittance of monolayer and bilayer graphene on $\mathrm{SiO}_{2}$ or $\mathrm{Si}$ wafers are, respectively, about 0.98 and 0.96 in the visible bandwidth. ${ }^{11,12}$ (iii) There is an optical-absorption window ${ }^{10,11,13}$ for radiation photon energy smaller than $0.2 \mathrm{eV}$. The width and depth of this window depend strongly on the temperature ${ }^{10}$ and carrier density (or gate voltage) in graphene samples. ${ }^{11}$ This interesting finding implies that graphene can also be used for infrared detection in ambient conditions. Further experimental investigation shows that graphene can have strong intraband and interband transitions which can be substantially modified through electrical gating, similar to the resistance tuning in graphene field-effect transistors. ${ }^{11,14}$ The optical and optoelectronic properties of graphene-based electronic systems have also been studied theoretically. ${ }^{15-17}$ Most of the theoretical results are in line with the main experimental findings but are valid for low temperatures and only electron scattering by acoustic phonons was considered. ${ }^{15}$ However, most of the experimental studies of the optical properties of graphene systems have been undertaken at relatively high temperatures and up to room temperature. ${ }^{10,11}$ In particular, the published theoretical work has not yet given a fully satisfactory explanation of the optical-absorption window, present in graphene in the infrared bandwidth, that is observed experimentally by different kinds of measurements. ${ }^{10,11,13}$ Therefore, in this study we examine how carriers in graphene respond to the applied radiation field in order to have a better understanding of the optoelectronic features of graphene, especially in the infrared wavelength regime. Here we would like to point out that graphene is a gapless electronic system in which optical phonon energy is about $196 \mathrm{meV} .{ }^{18}$ Hence, in contrast to a conventional semiconductor material which normally has a band gap much lager than the phonon energy, phonon scattering is expected to play an important role in affecting the electronic 
transitions accompanied by the emission and absorption of photons in graphene, especially in the infrared bandwidth. For the gapless graphene, the electrons in the valence band can gain the energy from the radiation field via optical absorption and be excited into the conduction band while the electrons in the conduction band can lose energy via emission of phonons and be relaxed into the valence band. Together with the fact that the electrons interact more strongly with phonons than with photons, carrier-phonon interaction is an important factor in affecting the interband electronic transitions and, therefore, in determining the optoelectronic properties of graphene at relatively high temperatures. In this work, we develop a systematic approach to calculate the electronic and optical coefficients of graphene in the presence of a radiation field. In such an approach, the electronphoton and electron-phonon couplings are included in the calculation and their consequences are examined in a consistently theoretical manner.

This paper is organized as follows. The theoretical approach to calculate the optoelectronic coefficients in graphene is developed in Sec. II. We evaluate the electronic transition rate induced by the interaction of electrons with photons and phonons. On this basis, we derive the massbalance and energy-balance equations from the Boltzmann equation. By solving these equations self-consistently, we obtain the optoelectronic coefficients. We present and discuss the numerical results from this study in Sec. III. In particular, we discuss the effect of the light radiation on photoinduced carrier densities, optical conductance, and transmission coefficient in an air-graphene-wafer system. Finally, we compared these results with those obtained experimentally and state our main conclusions in Sec. IV.

\section{THEORETICAL APPROACH}

\section{A. Electronic transition rate}

We consider a configuration where the graphene sheet is placed on the $(x, y)$ plane on top of a dielectric wafer such as $\mathrm{SiO}_{2} / \mathrm{Si}$ substrate. A light field is applied perpendicular to the graphene layer and is polarized linearly along the $x$ direction of the system. In the effective-mass approximation, a carrier (electron or hole) in a monolayer graphene can be described by Weyl's equation for a massless neutrino. ${ }^{19}$ The single-particle Hamiltonian that describes a Dirac quasiparticle in the $\pi$ bands near the $K$ point can be obtained from, e.g., the usual $\mathbf{k} \cdot \mathbf{p}$ approach. We assume that the system under study can be separated into the carriers of interest and the rest of the graphene crystal. Then the Hamiltonian that describes a carrier-photon-phonon system in graphene is $H(t)=H_{0}+H_{c o}(t)+H_{p h}+H_{c p}(t)$. Here,

$$
H_{0}=\gamma\left[\begin{array}{cc}
0 & \vec{k}_{x}-i \vec{k}_{y} \\
\vec{k}_{x}+i \vec{k}_{y} & 0
\end{array}\right]
$$

is the Hamiltonian for a Dirac quasiparticle and $\gamma=\hbar v_{F}$ is the band parameter with $v_{F}=10^{8} \mathrm{~cm} / \mathrm{s}$ being the Fermi velocity for a carrier in graphene, and $\vec{k}_{x}=-i \partial / \partial x$ is the momentum operator along the $x$ direction. In Eq. (1),

$$
H_{c o}(t)=\frac{\gamma e A(t)}{\hbar}\left[\begin{array}{ll}
0 & 1 \\
1 & 0
\end{array}\right]
$$

is the carrier-photon interaction Hamiltonian, $A(t)$ $=\left(F_{0} / \omega\right) \sin (\omega t)$ is the vector potential of the radiation field with $F_{0}$ and $\omega$ being, respectively, the electric field strength and frequency of the radiation field. Furthermore, $H_{p h}$ is the phonon Hamiltonian and

$$
H_{c p}(t)=V_{\mathbf{q}} a_{\mathbf{q}} e^{i\left(\mathbf{q} \cdot \mathbf{r}+\omega_{q} t\right)}+V_{\mathbf{q}}^{*} a_{\mathbf{q}}^{\dagger} e^{-i\left(\mathbf{q} \cdot \mathbf{r}+\omega_{q} t\right)}
$$

describes carrier interaction with 2D-like phonons, where $\mathbf{q}$ $=\left(q_{x}, q_{y}\right)$ is the phonon wave vector in the $(x, y)$ plane, $\left(a_{\mathbf{q}}^{\dagger}, a_{\mathbf{q}}\right)$ are the canonical conjugate coordinates of the phonon system, $V_{\mathbf{q}}$ is the carrier-phonon interaction coefficient, and $\omega_{q}$ is the phonon frequency in graphene. The effect of the radiation field has been included with the usual Coulomb gauge. The Schrödinger equation regarding $H_{0}$ can be solved analytically. ${ }^{19}$ For the case of a relatively weak radiation field and weak carrier-phonon interaction, $H_{c o}(t)$ and $H_{c p}(t)$ can be treated as perturbations. Using Fermi's golden rule, the first-order steady-state electronic transition rate is given by

$$
W_{\lambda \lambda^{\prime}}\left(\mathbf{k}, \mathbf{k}^{\prime}\right)=\sum_{\nu} W_{\lambda \lambda^{\prime}}^{\nu}\left(\mathbf{k}, \mathbf{k}^{\prime}\right)
$$

it is the probability for scattering of a carrier from a state $|\mathbf{k}, \lambda\rangle$ to a state $\left|\mathbf{k}^{\prime}, \lambda^{\prime}\right\rangle$ due to interaction with photons and phonons. Moreover, $\mathbf{k}=\left(k_{x}, k_{y}\right)$ is the wave vector of a carrier, $\nu$ refers to different scattering mechanisms, $\lambda=+1$ for an electron and $\lambda=-1$ for a hole. In Eq. (4), the rate

$$
\begin{aligned}
W_{\lambda \lambda^{\prime}}^{c o, \pm}\left(\mathbf{k}, \mathbf{k}^{\prime}\right)= & \frac{2 \pi}{\hbar}\left(\frac{e F_{0} \gamma}{2 \hbar \omega}\right)^{2} \frac{1+\lambda \lambda^{\prime} \cos (2 \phi)}{2} \\
& \times \delta_{\mathbf{k}^{\prime}, \mathbf{k}} \delta\left[E_{\lambda}(\mathbf{k})-E_{\lambda^{\prime}}\left(\mathbf{k}^{\prime}\right) \pm \hbar \omega\right]
\end{aligned}
$$

is induced by direct carrier-photon interaction via absorption (+ sign) and emission (- sign) of a photon with an energy $\hbar \omega, E_{\lambda}(\mathbf{k})=\lambda \gamma|\mathbf{k}|$ is the energy spectrum of a carrier, and $\phi$ is the angle between $\mathbf{k}$ and the $x$ axis. The rate

$$
\begin{aligned}
W_{\lambda \lambda^{\prime}}^{c p, \pm}\left(\mathbf{k}, \mathbf{k}^{\prime}\right)= & \frac{2 \pi}{\hbar}\left[\begin{array}{c}
N_{q} \\
N_{q}+1
\end{array}\right]\left|U_{\lambda \lambda^{\prime}}^{c p}(\mathbf{q}, \theta)\right|^{2} \delta_{\mathbf{k}^{\prime}, \mathbf{k}+\mathbf{q}} \\
& \times \delta\left[E_{\lambda}(\mathbf{k})-E_{\lambda^{\prime}}\left(\mathbf{k}^{\prime}\right) \pm \hbar \omega_{q}\right]
\end{aligned}
$$

is induced by carrier-phonon interaction, where $N_{q}$ $=\left(e^{\hbar \omega_{q} / k_{B} T}-1\right)^{-1}$ is the phonon occupation number, $\left|U_{\lambda \lambda^{\prime}}^{c p}(\mathbf{q}, \theta)\right|^{2}=\left|\left\langle\mathbf{k}^{\prime}, \lambda^{\prime}\left|V_{\mathbf{q}}\right| \mathbf{k}, \lambda\right\rangle\right|^{2}, \theta$ is the angle between $\mathbf{k}^{\prime}$ and $\mathbf{k}$, and the terms $N_{q}$ and $N_{q}+1$ correspond to the absorption and emission of a phonon, respectively. The rate

$$
\begin{aligned}
& W_{\lambda \lambda^{\prime}}^{c o p}\left(\mathbf{k}, \mathbf{k}^{\prime}\right)=\left.\frac{4 \pi}{\hbar}\left[\begin{array}{c}
\sqrt{N_{q}} \\
\sqrt{N_{q}+1}
\end{array}\right] \frac{e F_{0} \gamma}{2 \hbar \omega_{\mathbf{k}_{1}, \lambda_{1}}} \sum_{\lambda_{1} \lambda^{\prime}}(\mathbf{q}, \theta)\right|^{2} \\
& \times \sqrt{\left[1+\lambda \lambda_{1} \cos (2 \phi)\right] / 2} \delta_{\mathbf{k}, \mathbf{k}_{1}} \delta_{\mathbf{k}^{\prime}, \mathbf{k}_{1}+\mathbf{q}} \\
& \times \delta\left[E_{\lambda}(\mathbf{k})-E_{\lambda_{1}}\left(\mathbf{k}_{1}\right) \pm \hbar \omega+E_{\lambda_{1}}\left(\mathbf{k}_{1}\right)\right. \\
& \left.-E_{\lambda^{\prime}}\left(\mathbf{k}^{\prime}\right) \pm \hbar \omega_{q}\right]
\end{aligned}
$$

describes the coupled carrier-photon-phonon scattering via 
absorption and emission of both photons and phonons. This term also represents the indirect optical and electronic transition channels.

In this work, we consider carrier interaction with only optical phonons. It is known that at relatively high temperatures carriers in graphene interact more strongly with optical phonons. ${ }^{20}$ This coupling can result in a relatively large energy relaxation due to the inelastic nature of the scattering. Moreover, the published experimental results ${ }^{10,11,13}$ show that the optical-absorption window in graphene occurs near the photon-energy regime $\hbar \omega \sim 0.1 \mathrm{eV}$. This energy is much larger than the acoustic-phonon energy in graphene. As a result, at relatively high temperatures we can neglect the effect of the quasielastic scattering by acoustic phonons.

On the basis of the valence-force-field model, the coupling coefficient for carrier interaction with long-wavelength optical phonons in graphene is ${ }^{18,21}$

$$
V_{\mathbf{q}}^{\mu}=-g \gamma M_{\mathbf{q}}^{\mu} \text {. }
$$

Here $g=\left(\hbar \mathcal{B} / b^{2}\right) / \sqrt{2 \rho \hbar \omega_{0}}, \rho \simeq 6.5 \times 10^{-8} \mathrm{~g} / \mathrm{cm}^{2}$ is the areal density of the graphene sheet, $\omega_{0}=196 \mathrm{meV}$ the optical phonon frequency at the $\Gamma$ point, $\mathcal{B}=-d\left(\ln \gamma_{0}\right) / d(\ln b) \sim 2$ is a dimensionless parameter that relates to the change in the resonance integral $\gamma_{0}$ between nearest-neighbor carbon atoms, ${ }^{18}$ and $b=a / \sqrt{3}$ is the equilibrium bond length. Furthermore,

$$
M_{\mathbf{q}}^{l}=\left[\begin{array}{cc}
0 & -e^{-i \phi_{q}} \\
e^{i \phi_{q}} & 0
\end{array}\right] \text { and } M_{\mathbf{q}}^{t}=\left[\begin{array}{cc}
0 & i e^{-i \phi_{q}} \\
i e^{i \phi_{q}} & 0
\end{array}\right],
$$

describe the coupling with, respectively, longitudinal $(l)$ and transverse $(t)$ phonon modes, and $\phi_{q}$ is the angle between $\mathbf{q}$ and the $x$ axis. The carrier-phonon scattering matrix elements are

$$
\left|U_{\lambda \lambda^{\prime}}^{l}(\mathbf{q}, \mathbf{k})\right|=(g \gamma / \sqrt{2})\left[1-\lambda^{\prime} \lambda \cos \left(\phi+\phi^{\prime}-2 \phi_{q}\right)\right]^{1 / 2}
$$

and

$$
\left|U_{\lambda \lambda^{\prime}}^{t}(\mathbf{q}, \mathbf{k})\right|=(g \gamma / \sqrt{2})\left[1+\lambda^{\prime} \lambda \cos \left(\phi+\phi^{\prime}-2 \phi_{q}\right)\right]^{1 / 2} .
$$

\section{B. Balance equations}

In this work, we employ the semiclassical Boltzmann equation (BE) to study the response of the carriers in graphene to an applied radiation field. It is known that the Boltzmann equation is a powerful tool to study theoretically the linear and nonlinear responses of electrons in an electron gas system under the action of external driving ac and dc fields. In contrast to the Kubo formula based quantum theory which handles essentially the linear response, the BE can be used to study nonlinear transport and optical effects. ${ }^{22}$ In particular, the balance-equation approach based on the BE can be used to study nonequilibrium electronic transport and to calculate corresponding coefficients self-consistently such as the electron density and electron drift velocity in different states. ${ }^{23}$ In this work, we would like to use a consistent and tractable theory to calculate both photoexcited carrier density and photoinduced electron energy loss in graphene. The BEbased balance-equation approach is therefore a good theoret- ical option. It should be noted that although the momentumand energy-balance equations proposed by Lei and Ting ${ }^{24}$ based on quantum approach can handle the nonlinear transport of electrons under strong $\mathrm{dc}$ and/or intense ac fields, they can only be used to calculate the averaged electron drift velocity and electron energy loss rate. This approach cannot be applied to evaluate the electron density in different states. Furthermore, by employing the balance-equation approach on the basis of the $\mathrm{BE}$ to study graphene, ${ }^{20,25}$ we have already achieved a good agreement between theoretical results and experimental findings both qualitatively and quantitatively. The Boltzmann transport theory has also been applied to investigate graphene system by other authors. ${ }^{15}$ It was found ${ }^{15}$ that for a homogeneous graphene system with relatively high carrier density and long mean-free path (which is indeed the case for graphene), such a theory can lead to the same results as those obtained from quantum transport theory. ${ }^{16,17}$ For nondegenerate statistics, the BE can be written as

$$
\frac{\partial f_{\lambda}(\mathbf{k})}{\partial t}=g_{s} g_{v} \sum_{\lambda^{\prime}, \mathbf{k}^{\prime}, \nu}\left[F_{\lambda^{\prime} \lambda}^{\nu}\left(\mathbf{k}^{\prime}, \mathbf{k}\right)-F_{\lambda \lambda^{\prime}}^{\nu}\left(\mathbf{k}, \mathbf{k}^{\prime}\right)\right]
$$

where $g_{s}=2$ and $g_{v}=2$ account, respectively, for spin and valley degeneracy, $f_{\lambda}(\mathbf{k})$ is the momentum distribution function for a carrier in a state $|\mathbf{k}, \lambda\rangle$, and $F_{\lambda^{\prime} \lambda}^{\nu}\left(\mathbf{k}^{\prime}, \mathbf{k}\right)$ $=f_{\lambda^{\prime}}\left(\mathbf{k}^{\prime}\right)\left[1-f_{\lambda}(\mathbf{k})\right] W_{\lambda^{\prime} \lambda}^{\nu}\left(\mathbf{k}^{\prime}, \mathbf{k}\right)$. Because the radiation field has been included in the electronic transition rate, the force term induced by this field does not appear in the drift term on the left-hand side of the BE to avoid double counting. There is no simple and analytical solution to Eq. (7) with $W_{\lambda^{\prime} \lambda}\left(\mathbf{k}^{\prime}, \mathbf{k}\right)$ given by Eq. (4). In the present study we employ the usual balance-equation approach to solve the problem. For the first moment, the mass-balance equation (or rate equation) can be derived after operating with $g_{s} g_{v} \Sigma_{\mathbf{k}}$ on both sides of the $\mathrm{BE}$. The result is

$$
\frac{\partial n_{e}}{\partial t}=\frac{\partial n_{h}}{\partial t}=16 \sum_{\nu}\left(F_{-+}^{\nu}-F_{+-}^{\nu}\right)
$$

where $F_{\lambda^{\prime} \lambda}^{\nu}=\Sigma_{\mathbf{k}^{\prime}, \mathbf{k}} F_{\lambda^{\prime} \lambda}^{\nu}\left(\mathbf{k}^{\prime}, \mathbf{k}\right)$ and $n_{e}$ and $n_{h}$ are, respectively, the electron and hole densities in different bands. This equation implies that only interband scattering (i.e., $\lambda^{\prime} \neq \lambda$ ) can alter the number of carries in a band of the graphene system. It also reflects the fact that the change in the electron number in the conduction band equals that of the hole number in the valence band, namely, this equation expresses the charge number conservation in the system.

For the second moment, the energy-balance equations can be derived by operating with $\sum_{\mathbf{k}, \lambda} E_{\lambda}(\mathbf{k})$ on both sides of the BE. From the energy-balance equation, we obtain the energy transfer rate for a carrier, $P_{\lambda}=\Sigma_{\mathbf{k}} E_{\lambda}(\mathbf{k}) \partial f_{\lambda}(\mathbf{k}) / \partial t$, and the total energy transfer rate of the system is $P=P_{+}+P_{-}=P_{p h}$ $-P_{o p}$, where

$$
P_{o p}=4 \hbar \omega \sum_{\lambda^{\prime}, \lambda}\left(F_{\lambda \lambda^{\prime}}^{c o,+}-F_{\lambda \lambda^{\prime}}^{c o,-}+F_{\lambda \lambda^{\prime}}^{c o p}\right)
$$

is the energy transfer rate induced by optical absorption and emission via direct and indirect transition channels, and 
$F_{\lambda \lambda^{\prime}}^{c o, \pm}$ describes the absorption $(+)$ and emission (-) of photons. Further,

$$
P_{p h}= \pm 4 \sum_{\lambda^{\prime}, \lambda} \hbar \omega_{q}\left(F_{\lambda \lambda^{\prime}}^{c p, \pm}+F_{\lambda \lambda^{\prime}}^{c o p}\right)
$$

is the energy transfer rate induced by emission or absorption of optical phonons. In the steady state, $P=0$ and $P_{o p}=P_{p h}$ gives an energy conservation law, namely, the carriers in the system gain energy from the radiation field via absorption of photons and phonons and lose energy via emission of optical phonons and photons.

For coupled carrier-photon-phonon scattering via absorption and emission of photons and optical phonons, Eqs. (5) and (6) give

$$
\begin{aligned}
W_{\lambda \lambda^{\prime}}^{c o p}\left(\mathbf{k}, \mathbf{k}^{\prime}\right)= & \frac{4 \pi}{\hbar}\left[\frac{\sqrt{N_{0}}}{\sqrt{N_{0}+1}}\right] \frac{e F_{0} g \gamma^{2}}{2 \hbar \omega}(\cos \Phi+\sin \Phi) \\
& \times(\cos \phi+\sin \phi) \delta_{\mathbf{k}^{\prime}, \mathbf{k}+\mathbf{q}} \delta\left[E_{\lambda}(\mathbf{k})-E_{\lambda^{\prime}}\left(\mathbf{k}^{\prime}\right)\right. \\
& \left.+\hbar \omega \pm \hbar \omega_{q}\right]
\end{aligned}
$$

with $\Phi=\left(\phi+\phi^{\prime}-2 \phi_{q}\right) / 2$. As a result, we explicitly obtain

$$
F_{+-}^{c o p}=F_{-+}^{c o p}=0 .
$$

This result implies that in graphene the coupled carrierphoton-phonon interaction via coupling with longwavelength optical phonons does not contribute to electronic transitions. In the steady state, i.e., for $d n_{e} / d t=d n_{h} / d t=0$, the mass-balance equation becomes

$$
F_{-+}^{c o,+}+F_{-+}^{c p,-}=F_{+-}^{c p,+}+F_{+-}^{c o,-},
$$

which reflects the fact that electrons pumped from the valence band into the conduction band are balanced by those relaxed from the conduction band into the valence band. Furthermore, the energy transfer rate induced by optical absorption and emission in the steady state is

$$
P_{o p}=4 \hbar \omega \sum_{\lambda^{\prime}, \lambda}\left[F_{\lambda \lambda^{\prime}}^{c o,+}-F_{\lambda \lambda^{\prime}}^{c o,-}\right]
$$

When a graphene sheet is subjected to a radiation field, electrons in the occupied states, e.g., in the lower energy $\lambda=-$ band, are excited into the empty states, e.g., of the higher energy $\lambda=+$ band so that an optical absorption occurs.

One of the advantages of the balance-equation approach is that we can avoid the difficulties to solve the BE directly and instead use a certain form of the carrier distribution function to calculate the physical quantities. Here we use the FermiDirac type of statistical energy distribution as approximately the momentum distribution for a carrier, i.e., $f_{\lambda}(\mathbf{k})$ $\simeq f_{\lambda}(\lambda \gamma k)$, with $f_{\lambda}(x)=\left[1+e^{\left(x-\mu_{\lambda}^{*}\right) / k_{B} T}\right]^{-1}$, where $\mu_{\lambda}^{*}$ is the quasi Fermi energy (or quasichemical potential) for electrons or holes in the presence of the radiation field. For carrierphoton interaction via interband and intraband transition channels, we have $F_{+-}^{c o,+}=F_{-+}^{c o,-}=F_{++}^{c o,-}=F_{--}^{c o,-}=0$,

$$
F_{-+}^{c o,+}=\frac{e^{2} F_{0}^{2}}{32 \hbar^{2} \omega} f_{-}\left(-\frac{\hbar \omega}{2}\right)\left[1-f_{+}\left(\frac{\hbar \omega}{2}\right)\right]
$$

is for optical absorption from the valence band to the conduction band,

$$
F_{+-}^{c o,-}=\frac{e^{2} F_{0}^{2}}{32 \hbar^{2} \omega} f_{+}\left(\frac{\hbar \omega}{2}\right)\left[1-f_{-}\left(-\frac{\hbar \omega}{2}\right)\right]
$$

is for optical emission from the conduction band to the valence band, and

$$
F_{\lambda \lambda}^{c o,+}=\frac{e^{2} F_{0}^{2}}{8 \pi \hbar^{4} \omega^{3}} \frac{\omega \tau_{\lambda}}{1+\left(\omega \tau_{\lambda}\right)^{2}} \int_{0}^{\infty} d E E f_{\lambda}(\lambda E)\left[1-f_{\lambda}(\lambda E)\right]
$$

is induced by intraband optical absorption in the conduction band $(\lambda=1)$ and valence band $(\lambda=-1)$ with $\tau_{\lambda}$ being the energy relaxation time for an electron or a hole in different bands. In fact, this term is caused by the usual free-carrier absorption channels. The energy relaxation time is used to describe the broadening of the scattering states, which can result in a spectrum structure for the intraband optical absorption. It should be noted that for intraband free-carrier absorption, the momentum conservation law still holds [i.e., for optical transitions the electron momentum at initial and final states must be the same during a scattering event, as given by Eq. (4)]. However, in the presence of the external driving fields such as the radiation fields and of the scattering centers such as impurities and phonons, the scattering states are damped and broadened. As a result, the $\delta$ function in Eq. (4) for intraband optical transition can be replaced through Poisson Kernel: $\delta(E) \rightarrow\left(E_{\lambda} / \pi\right)\left(E^{2}+E_{\lambda}^{2}\right)^{-1}$ with $E_{\lambda}=\hbar / \tau_{\lambda}$ being the energy broadening of the states.

For carrier-phonon interaction via different transition channels, we have $F_{+-}^{c p,-}=F_{-+}^{c p,+}=0$,

$$
\begin{aligned}
F_{+-}^{c p,+}= & \frac{g^{2}\left(N_{0}+1\right)}{2 \pi \hbar \gamma^{2}} \int_{0}^{\hbar \omega_{0}} d x x\left(\hbar \omega_{0}-x\right) \\
& \times f_{+}(x)\left[1-f_{-}\left(x-\hbar \omega_{0}\right)\right]
\end{aligned}
$$

is for phonon emission and corresponding electronic transition from the conduction band to the valence band, with $N_{0}=\left[e^{\hbar \omega_{0} / k_{B} T}-1\right]^{-1}$ and $\omega_{q} \rightarrow \omega_{0}$ a constant at the $\Gamma$ point for long-wavelength optical phonons, and

$$
F_{-+}^{c p,-}=\frac{g^{2} N_{0}}{2 \pi \hbar \gamma^{2}} \int_{0}^{\hbar \omega_{0}} d x x\left(\hbar \omega_{0}-x\right) f_{-}(-x)\left[1-f_{+}\left(\hbar \omega_{0}-x\right)\right]
$$

is induced by the absorption of phonons and corresponding electronic transition from the valence band to the conduction band.

\section{Photoinduced carriers}

We now consider a graphene layer in which the conducting carriers are electrons (or a positive gate voltage is applied) in the absence of the radiation field. When a light field is applied to the system, the electrons in the valence band are 
excited into the conduction band so that photoexcited carriers can be induced. If $n_{0}$ is the electron density in the absence of the radiation field (or dark density) at $F_{0}=0$, the electron density at $F_{0} \neq 0$ is $n_{e}=n_{0}+\Delta n_{e}$. On account of charge number conservation, we have $\Delta n_{e}=n_{h}$, the hole density in the presence of radiation field. Thus, we get

$$
n_{e}=n_{0}+n_{h}
$$

with

$$
n_{e}=\frac{2}{\pi \gamma^{2}} \int_{0}^{\infty} \frac{d x x}{e^{\left(x-\mu_{e}^{*}\right) / k_{B} T}+1}
$$

and

$$
n_{h}=n_{e}-n_{0}=\frac{2}{\pi \gamma^{2}} \int_{0}^{\infty} \frac{d x x}{e^{\left(x+\mu_{h}^{*}\right) / k_{B} T}+1} .
$$

With the mass-balance equation given by Eq. (12) and the requirement of the charge number conservation shown as Eq. (19) we can determine the quasichemical potentials $\mu_{\lambda}^{*}$ for electrons and holes. Then the electron density $n_{e}$ and hole density $n_{h}$ can be obtained in the presence of the radiation field $F_{0}$. We notice that this approach can also be applied to $p$-type graphene samples, when a negative gate voltage is applied so that the conducting carriers are holes in the valence band in the dark.

\section{Optical conductance and transmission}

With the obtained carrier chemical potential $\mu_{\lambda}^{*}$, we can calculate $F_{\lambda \lambda^{\prime}}^{c o, \pm}$. From the carrier energy transfer rate induced by optical absorption, described by Eq. (13), we can calculate the optical conductance $\sigma(\omega)$ for graphene using the expression $^{26}$

$$
\sigma(\omega)=2 P_{o p} / F_{0}^{2}=8 \hbar \omega \sum_{\lambda, \lambda^{\prime}}\left(F_{\lambda, \lambda^{\prime}}^{c o,+}-F_{\lambda, \lambda^{\prime}}^{c o,-}\right) / F_{0}^{2} .
$$

Moreover, the transmission coefficient for a device with a graphene layer on top of a substrate, namely, for an airgraphene-wafer system, is given by ${ }^{17}$

$$
T(\omega)=\sqrt{\frac{\epsilon_{2}}{\epsilon_{1}}} \frac{4\left(\epsilon_{1} \epsilon_{0}\right)^{2}}{\left|\left(\sqrt{\epsilon_{1} \epsilon_{2}}+\epsilon_{1}\right) \epsilon_{0}+\sqrt{\epsilon_{1}} \sigma(\omega) / c\right|^{2}},
$$

where $\epsilon_{1} \simeq 1$ for air, $\epsilon_{2}$ is the effective high-frequency dielectric constant of the substrate, and $\epsilon_{0}$ and $c$ are, respectively, the dielectric constant and the speed of light in vacuum. It indicates that the light transmittance of the graphene layer in an air-graphene-substrate system depends on the dielectric constant of the substrate material. Moreover, a substrate with a larger dielectric constant can result in a smaller light transmittance for the graphene layer.

\section{NUMERICAL RESULTS AND DISCUSSIONS}

In the numerical calculations, we consider a typical air-graphene- $\mathrm{SiO}_{2} /$ wafer system. Thus, $\epsilon_{1} \simeq \epsilon_{0}=1$ and $\epsilon_{2}$ $\simeq 2$.0. The effect of the dielectric mismatch between the

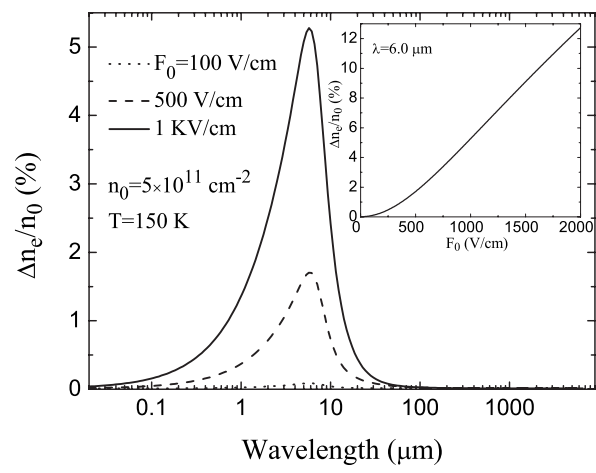

FIG. 1. Photoexcited electron density $\Delta n_{e}$ as a function of the radiation wavelength, at temperature $T=150 \mathrm{~K}$ and a fixed dark electron density $n_{0}=5 \times 10^{11} \mathrm{~cm}^{-2}$, for different strengths of the radiation field $F_{0}$. The inset shows $\Delta n_{e}$ as a function of $F_{0}$ at a fixed radiation wavelength $\mathcal{L}=6.0 \mu \mathrm{m}$. Note that $\Delta n_{e}$ is equal to $n_{h}$, the photoinduced hole density.

graphene layer and the $\mathrm{SiO}_{2}$ substrate has been taken into account using the image charge method. ${ }^{25}$ Furthermore, it has been obtained experimentally ${ }^{27}$ that in graphene the energy relaxation time is about $\tau_{\lambda} \sim 1$ ps for high-density samples. Thus, we take $\tau_{\lambda} \sim 1$ ps in the calculation for freecarrier absorption. A typical electric field strength of the radiation field $F_{0}=500 \mathrm{~V} / \mathrm{cm}$ is used in most of the calculations.

\section{A. Photoinduced carrier densities}

In Fig. 1, we show the dependence of the photon-excited electron density $\Delta n_{e}$ on the radiation wavelength $(\mathcal{L})$ and radiation intensity for a fixed dark electron density $n_{0}$ and a temperature $T=150 \mathrm{~K} . \Delta n_{e}$ vs the strength of the radiation field $F_{0}$ is also shown in the inset for fixed radiation wavelength $\mathcal{L}=6.0 \mu \mathrm{m}$. It should be noted that $\Delta n_{e}=n_{h}$ is also the photoinduced hole density for a $n$-type graphene in the dark. As expected, the photoinduced carrier densities increase with radiation intensity $I \sim F_{0}^{2}$. For a typical radiation intensity with $F_{0}$ about $500 \mathrm{~V} / \mathrm{cm}$, several percents of photoinduced electron density can be achieved in graphene, similar to photoexcited carriers in conventional semiconductors. We notice that when the radiation wavelength is about $\mathcal{L} \sim 6 \mu \mathrm{m} \Delta n_{e}$ is maximum. Because the optical phonon wavelength in graphene is about $6 \mu \mathrm{m}$, the peak of $\Delta n_{e}$ appears at about this wavelength. For $\mathcal{L}>6 \mu \mathrm{m}, \Delta n_{e}$ decreases sharply with increasing $\mathcal{L}$. These results indicate that in graphene the photoinduced carrier densities can be observed clearly in the infrared bandwidth.

In Fig. 2, we show the photoexcited electron density $\Delta n_{e}$ as a function of the radiation wavelength, at fixed dark electron density and radiation intensity, for different temperatures. We see that the photoexcited carrier densities are very sensitive to temperature. $\Delta n_{e}$ decreases quickly with increasing temperature. Such a feature is typical when scattering by optical phonons is present. With increasing temperature, the phonon occupation number $N_{0}=\left[e^{\hbar \omega_{0} / k_{B} T}-1\right]^{-1}$ increases sharply so that a stronger phonon scattering occurs. The strong phonon scattering can bring electrons from the con- 




FIG. 2. Photoexcited electron density $\Delta n_{e}$ as a function of the radiation wavelength, for fixed dark electron density $n_{0}=1$ $\times 10^{12} \mathrm{~cm}^{-2}$ and radiation intensity $F_{0}=500 \mathrm{~V} / \mathrm{cm}$, at different temperatures.

duction band to the valence band via phonon emission. As a consequence, at relatively high temperatures less photoexcited electrons remain in the conduction band. Accordingly, $\Delta n_{e}$ decreases with increasing temperature. These results suggest that phonon scattering is an important mechanism in affecting the photoexcited carrier densities in graphene.

In Fig. 3, $\Delta n_{e}$ is plotted as a function of the radiation wavelength, for $T=150 \mathrm{~K}$ and $F_{0}=500 \mathrm{~V} / \mathrm{cm}$, for different dark electron densities. The main feature in Fig. 3 is that the photoinduced carrier density ratio $\Delta n_{e} / n_{0}$ decreases with increasing dark electron density $n_{0}$. At relatively weak levels of light excitation $\Delta n_{e}$ changes only by several percents (see also Figs. 1 and 2). This implies that the change in the quasiFermi levels in the system due to a weak excitation is not very significant. A larger $n_{0}$ means a higher Fermi level in the conduction band in the absence of the radiation field. As we know, the electrons make transitions mainly from the occupied states to the empty states in an electron gas system. For larger $n_{0}$ only the higher energy states above the Fermi level are available for photoinduced electrons to be excited to. Thus, larger photon energy (or shorter wavelength radiation) is required to excite electrons from the valence band to the conduction band. On the other hand, because of the linear energy spectrum for carriers in graphene, larger energy states correspond to larger momentum states in the conduction band. Since the electron-phonon scattering alters both the

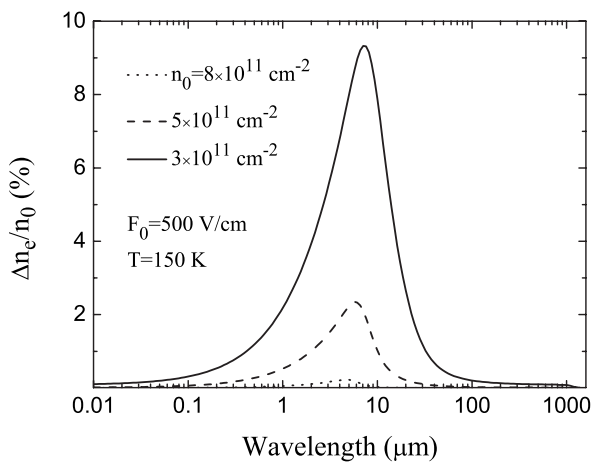

FIG. 3. $\Delta n_{e}$ as a function of the radiation wavelength at a temperature $T=150 \mathrm{~K}$ and fixed strength of the radiation field $F_{0}$ $=500 \mathrm{~V} / \mathrm{cm}$, for different dark electron densities. energy and momentum, as required by the corresponding energy and momentum conservation laws, larger momentum states can result in stronger electron-phonon coupling. Again, the strong phonon scattering can reduce the photoinduced electron density in the conduction band. Therefore, the densities shown in Fig. 3 are, at least partly, the result of electron-phonon interaction. Moreover, it should be noted that the dark carrier density in graphene can be modulated effectively by applying a gate voltage..$^{11,14}$ Hence, the densities of photoinduced carriers can be modulated electrically as well.

The main results from Figs. 1-3 are as follows. (i) The photoinduced electron density increases with radiation wavelength for $\mathcal{L}<6.0 \mu \mathrm{m}$. (ii) The peak of the photoexcited electron density can be observed around $\mathcal{L} \sim 6.0 \mu \mathrm{m}$, which corresponds to the optical phonon energy $\hbar \omega_{0}=196 \mathrm{meV}$ in graphene. (iii) The photoinduced electron density decreases rapidly with increasing radiation wavelength for $\mathcal{L}$ $>6.0 \mu \mathrm{m}$. When a graphene sample is subjected to a radiation field and the electron-phonon interaction is present, electrons in the valence band can gain energy from the radiation field through optical absorption and be excited into the conduction band while electrons in the conduction band can lose energy via emission of phonons and be relaxed into the valence band. The balance of these two competing processes results in the photoinduced carriers in the system in the steady state. As we know, the optical phonon emission occurs when the electron energy is larger than the optical phonon energy. ${ }^{28}$ For $\mathcal{L}<6.0 \mu \mathrm{m}$, electrons located around the top of the valence band can gain a photon energy $\hbar \omega$ $>\hbar \omega_{0}$ via optical absorption and be excited into the conduction band. These electrons can lose energy $\hbar \omega_{0}$ through emission of optical phonons and be relaxed into the empty states of the valence band. This is why photoexcited carrier densities increase with the radiation wavelength for $\mathcal{L}$ $<6.0 \mu \mathrm{m}$. At relatively long-wavelength radiations for $\mathcal{L}$ $>6.0 \mu \mathrm{m}$, the electrons around the top of the valence band gain energy $\hbar \omega<\hbar \omega_{0}$ and are excited into the conduction band. However, when the electron energy is less than the optical phonon energy electronic transitions via phonon emission are much less likely. ${ }^{28}$ Thus, for $\hbar \omega<\hbar \omega_{0}$ the electrons in the conduction band are less likely to be relaxed into the valence band though electron-phonon coupling. This is the main reason why the photoexcited carrier densities decrease sharply with increasing radiation wavelength for $\hbar \omega$ $<\hbar \omega_{0}$. When the photon energy is close to the optical phonon energy $\hbar \omega \sim \hbar \omega_{0}$, interband electronic transitions can occur through a process in which the electrons gain a photon energy $\hbar \omega$ from the radiation field and lose a phonon energy $\hbar \omega_{0}$ via phonon emission. Thus, a resonant transition occurs in graphene. Such a mechanism is electrically equivalent to the electro-phonon resonance effect ${ }^{28}$ proposed previously by us. As a result, the strongest interband electronic transitions can occur for $\hbar \omega \sim \hbar \omega_{0}$. That is, a peak of the photoinduced carrier density can be observed in graphene when the radiation frequency is close to the optical phonon frequency. The theoretical results discussed here indicate that in gapless graphene the scattering by optical phonons plays an important role in affecting photoexcited carrier densities. 


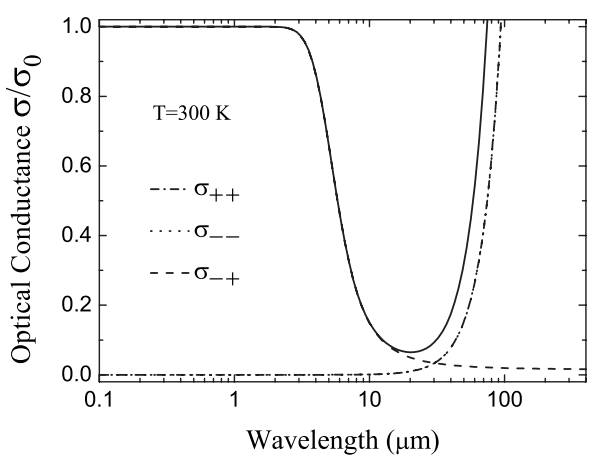

FIG. 4. Contribution to the total optical conductance (solid line) from different transition channels at room temperature for a fixed dark electron density $n_{0}=1 \times 10^{12} \mathrm{~cm}^{-2}$. Here $\sigma_{0}=e^{2} /(4 \hbar)$ and $\sigma_{-+}$ (dashed curve) is for transition from valence band (-) to conduction band $(+)$. The curves for intraband transitions, $\sigma_{++}$and $\sigma_{--}$, coincide roughly.

\section{B. Optical conductance and transmission}

In Fig. 4, we show the contributions from different transition channels to the optical conductance $\sigma(\omega)$ or optical absorption for a fixed dark electron density $n_{0}$ at room temperature. We notice the following features. (i) Interband transitions contribute to the optical absorption in the shortwavelength regime $(\mathcal{L}<3 \mu \mathrm{m})$ whereas intraband transitions give rise to the long-wavelength optical absorption. (ii) The optical absorption varies very little upon varying the radiation frequency in the short-wavelength regime $(\mathcal{L}<3 \mu \mathrm{m})$, whereas the optical conductance or absorption coefficient depends strongly on the radiation wavelength in the long-wavelength regime $(\mathcal{L}>3 \mu \mathrm{m})$. (iii) The optical conductance in the short-wavelength regime is a universal value $\sigma_{0}=e^{2} /(4 \hbar)$ in monolayer graphene as discovered experimentally. ${ }^{10-12}$ (iv) More interestingly, there is an infrared absorption window in the 4-100 $\mu \mathrm{m}$ wavelength range. As expected, interband transitions require larger photon energy. Intraband transitions, which are caused by the usual free-carrier absorption, occur under low photon-energy radiation. It is a common feature for free-carrier absorption that the strength of the optical absorption increases rapidly with radiation wavelength. ${ }^{29}$ We find that the opticalabsorption window observed experimentally ${ }^{10,11,13}$ is induced by the competing absorption channels due to interband and intraband scattering events in graphene. This can explain and reproduce recent experimental findings. ${ }^{10,11,13}$

The interesting features of optical absorption in graphene can be understood with the help of Fig. 5. When the radiation field is absent, there is a single Fermi level (or chemical potential) in the conduction band in a $n$-type graphene sample (or in the presence of a positive gate voltage). In this case all states below $\mu_{e}^{*}$ are occupied by electrons as shown in Fig. 5(a). When a radiation field is applied to the system [see Fig. 5(b)], the electrons in the valence band can gain energy from the radiation field and be excited into the conduction band via absorption of photons. Thus, the electron density in the conduction band increases and so does the quasi Fermi level $\mu_{e}^{*}$ for electrons. Meanwhile, the holes are left in the valence band and a quasi-Fermi level $\mu_{h}^{*}$ is estab-



FIG. 5. (a) A graphene system in the absence of the radiation field $\left(F_{0}=0\right)$. The conducting carriers are electrons with a Fermi energy $\mu_{e}^{*}$ in the conduction band. The hatched area shows the occupied states. (b) Optical-absorption channels in the presence of a radiation field $\left(F_{0} \neq 0\right)$. Here $\mu_{e}^{*}$ and $\mu_{h}^{*}$ are the quasi-Fermi energies for, respectively, electrons and holes and there are three optical-absorption channels: $\alpha_{-+}, \alpha_{++}$, and $\alpha_{--} . \hbar \omega_{0}$ is the energy of an emitted optical phonon.

lished in this band for them. As shown in Fig. 5(b), in the presence of a radiation field the intraband electronic transition accompanied by the absorption of photons can occur not only in the conduction band via the $\alpha_{++}$channel but also in the valence band via the $\alpha_{--}$channel. The intraband transitions are a direct consequence of the broadening of the scattering states in the conduction and valence bands. At the same time, the electrons in the conduction band can lose energy via emission of optical phonons and relax into the valence band. The electrons in the valence band can also gain energy by absorption of optical phonons and be excited into the conduction band due to the inelastic character of the electron-phonon scattering, although such a process is much weaker than that for phonon emission. Because graphene is a gapless semiconductor, the electrons in the valence band can be more easily excited into the conduction band via optical absorption and those in the conduction band can be easily relaxed into the valence band via phonon emission, in contrast to a conventional semiconductor. Thus, there is a strong interband optical and electronic transition channel [i.e., $\alpha_{-+}$ in Fig. 5(b)] in graphene. Since optical absorption and phonon emission events describe transitions from occupied states to empty states, the intraband transitions require less photon energy whereas a relatively larger photon energy is needed for interband transitions. Consequently, an opticalabsorption window can be induced through different energy requirements for intertransition and intratransition channels.

In Fig. 6, we show the optical conductance $\sigma$ and transmission coefficient $T(\omega)$ as a function of the radiation wavelength, at fixed dark electron density $n_{0}$, for different temperatures. As can be seen, in the short-wavelength regime $\mathcal{L}<3 \mu \mathrm{m}$, both $\sigma$ and $T(\omega)$ depend very little on the radiation wavelength. This confirms that $\sigma$ does not depend on temperature under short-wavelength radiation in graphene. The corresponding transmission coefficient $T(\omega)$ is about $0.97-0.98$ in the short-wavelength regime and agrees quantitatively with the experimental data. ${ }^{12}$ In the long- 


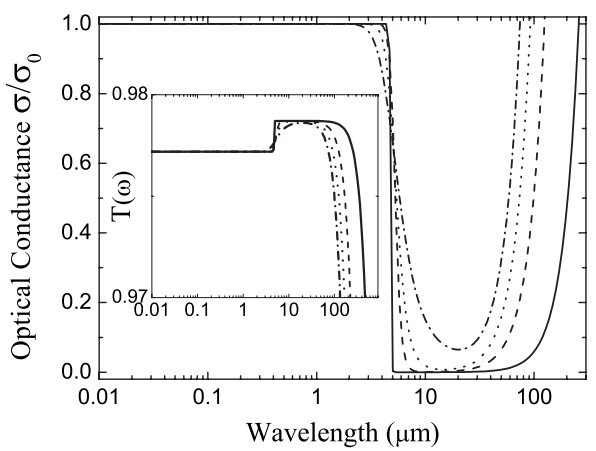

FIG. 6. Optical conductance and transmission coefficient (inset) as a function of the radiation wavelength, at a fixed dark electron density $n_{0}=1 \times 10^{12} \mathrm{~cm}^{-2}$, for different temperatures $T=10 \mathrm{~K}$ (solid curve), $77 \mathrm{~K}$ (dashed curve), $150 \mathrm{~K}$ (dotted curve), and $300 \mathrm{~K}$ (dotted-dashed curve).

wavelength regime, in which the optical-absorption window can be observed, both the optical conductance and light transmittance depend sensitively on the temperature, which is in line with the experimental findings..$^{10}$ It should be noted that for fixed electron and hole densities, the quasichemical potential for electrons/holes decreases/increases with increasing temperature. Thus, due to the Pauli blockade effect, ${ }^{30}$ a blueshift of the optical-absorption window with decreasing temperature can be observed as shown in Fig. 6. We note that the strength of the optical absorption is proportional to the optical conductance. Therefore, the height of the optical-absorption window decreases with increasing temperature. We find that a wider and deeper optical-absorption window and a sharper cutoff of the optical absorption at the window edges can be observed at lower temperatures. These theoretical results can be used to explain the strong dependence of the optical conductance on temperature in the infrared bandwidth that was observed experimentally. ${ }^{10,12}$

The optical conductance $\sigma$ and transmission coefficient $T(\omega)$ are shown in Fig. 7 as a function of the radiation wavelength at room temperature for different dark electron densities $n_{0}$. We note that the Fermi level for electrons becomes higher with increasing dark electron density. A higher Fermi level for electrons implies that the empty states in the conduction band have higher energies because of the linear

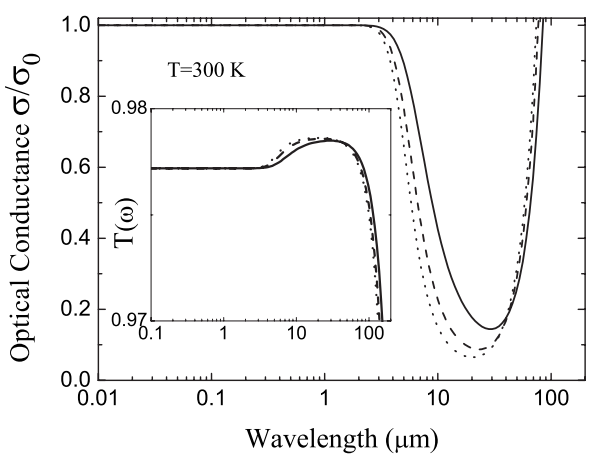

FIG. 7. Optical conductance and transmission coefficient (inset) as a function of the radiation wavelength, at room temperature, for different dark electron densities $n_{0}=5 \times 10^{11} \mathrm{~cm}^{-2}$ (solid curve), $8 \times 10^{11} \mathrm{~cm}^{-2}$ (dashed curve), and $1 \times 10^{12} \mathrm{~cm}^{-2}$ (dotted curve). shape of the energy spectrum for graphene. Since the optical transitions occur mainly via exciting electrons from occupied states to the empty states, a higher Fermi level corresponds to a higher transition energy. Thus, a blueshift of the opticalabsorption window can be observed in graphene samples with larger dark electron densities as shown in Fig. 7. This blueshift, with increasing gate voltage, has been observed experimentally. ${ }^{11}$ Because in graphene the dark electron density increases almost linearly with increasing gate voltage, ${ }^{4}$ the theoretical results shown in Fig. 7 are in agreement with these experimental findings obtained from optical-absorption measurements. ${ }^{11}$ Furthermore, we find that the height of the absorption window increases with dark electron density and a sharper cutoff of the optical absorption at the window edges can be observed for larger electron densities. These theoretical results suggest that the width and height of the infrared absorption window in graphene can be controlled by applying a gate voltage. This feature can be utilized for making graphene-based and frequency-tunable infrared optoelectronic devices.

\section{CONCLUSIONS}

In this work, we have examined theoretically the effect of optical phonon scattering on the optoelectronic properties of graphene. On the basis of the Boltzmann equation approach, we have derived the mass-balance and energy-balance equations for graphene in the presence of a linearly polarized radiation field and of the electron-photon-phonon coupling. By solving these equations self-consistently, we have been able to determine the photoexcited carrier densities and optoelectronic coefficients, such as the optical conductance and light transmittance, for an air-graphene-wafer system. In particular, we have investigated the dependence of the photoinduced carrier densities, optical conductance, and transmission coefficient on intensity and wavelength of the radiation field, along with those on temperature and dark electron density. The main conclusions we have obtained from this work are summarized as follows.

Because graphene is a gapless electronic system, electronphonon interaction is an important mechanism in affecting the electronic transitions via both intraband and interband transition channels. In graphene the electrons in the valence band can gain the energy from the radiation field via optical absorption and be excited into the conduction band. At the same time, the electrons in the conduction band can lose energy via emission of phonons and be relaxed into the valence band. Thus, electron-phonon scattering can affect strongly the interband transition in contrast to conventional semiconductors. As a result, the electron-photon-phonon interaction is a major scattering mechanism to determine photoinduced carrier densities and optoelectronic properties of graphene.

In the presence of a radiation field, the photoexcited carrier densities in graphene first increase and then decrease with increasing radiation wavelength. The largest carrier densities caused by light radiation can be observed when the radiation photon energy equals to the optical phonon energy of graphene. Such resonant transitions are electrically 
equivalent to the electro-phonon resonance effect observed in conventional two-dimensional electron-gas systems. The photoexcited carrier densities depend strongly on the radiation intensity and frequency, temperature, and dark carrier density.

In the short-wavelength regime $(\mathcal{L}<3 \mu \mathrm{m})$, the universal optical conductance $\sigma_{0}=e^{2} /(4 \hbar)$ and light transmittance $T_{0}$ $\sim 0.98$ can be achieved for an air-graphene- $\mathrm{SiO}_{2} /$ wafer system. The optical conductance and transmission coefficient depend very little on temperature and dark electron density. These results agree with other theoretical works and with experimental findings.

We have found that there is an optical-absorption window in the radiation wavelength range 4-100 $\mu \mathrm{m}$. This infrared absorption window is induced by different transition energies required for interband and intraband optical absorption in the presence of the Pauli blockade effect. The depth and width of such an absorption window depend sensitively on the temperature and dark electron density in the sample due to the presence of a free-carrier absorption in this radiation wavelength regime. A prominent cutoff of the optical absorption can be observed at the edges of the window at lower temperatures and/or larger dark electron densities. These results can explain why experimentally the optical-absorption win- dow can be measured under long-wavelength radiation and why experimentally a blueshift of such a window can be observed when increasing the gate voltage.

The results obtained from this study indicate that in addition to the excellent optical properties of graphene in the visible regime, i.e., universal optical conductance, high light transmittance, etc., graphene can exhibit interesting and important features in the midinfrared bandwidth, such as the optical-absorption window. The width and the depth of this window can be tuned by varying the dark carrier density via, e.g., applying a gate voltage. This implies that graphene can be used as a frequency-tunable optoelectronic device operating in the midinfrared bandwidth at room temperature for various applications. Together with the relevant phenomena discovered by very recent experimental work, ${ }^{10,11,13}$ we hope that the present work sheds some light on the application of graphene not only as a visible optoelectronic device but also as an infrared device in ambient conditions.

\section{ACKNOWLEDGMENTS}

This work was supported by the Chinese Academy of Sciences, National Natural Science Foundation of China, and Department of Science and Technology of Yunnan Province. *wenxu_issp@yahoo.cn

${ }^{1}$ K. S. Novoselov, A. K. Geim, S. V. Morozov, D. Jiang, Y. Zhang, S. V. Dubonos, I. V. Grigoreva, and A. A. Firsov, Science 306, 666 (2004).

${ }^{2}$ Y. B. Zhang, Y. W. Tan, H. L. Stormer, and P. Kim, Nature (London) 438, 201 (2005).

${ }^{3}$ C. Berger, Z. Song, X. Li, X. Wu, N. Brown, C. Naud, D. Mayou, T. Li, J. Hass, A. N. Marchenkov, E. H. Conrad, P. N. First, and W. A. de Heer, Science 312, 1191 (2006).

${ }^{4}$ K. S. Novoselov, A. K. Geim, S. V. Morozov, D. Jiang, M. I. Katsnelson, I. V. Grigorieva, S. V. Dubonos, and A. A. Firsov, Nature (London) 438, 197 (2005).

${ }^{5}$ E. V. Castro, K. S. Novoselov, S. V. Morozov, N. M. R. Peres, J. M. B. Lopes dos Santos, J. Nilsson, F. Guinea, A. K. Geim, and A. H. Castro Neto, Phys. Rev. Lett. 99, 216802 (2007).

${ }^{6}$ J. González and E. Perfetto, J. Phys.: Condens. Matter 20, 145218 (2008).

${ }^{7}$ Y.-M. Lin, K. A. Jenkins, A. Valdes-Garcia, J. P. Small, D. B. Farmer, and P. Avouris, Nano Lett. 9, 422 (2009).

${ }^{8}$ G. Eda, G. Fanchini, and M. Chhowalla, Nat. Nanotechnol. 3, 270 (2008).

${ }^{9}$ H. Hogan, Photonics Spectra 42, 19 (2008).

${ }^{10}$ A. B. Kuzmenko, E. van Heumen, F. Carbone, and D. van der Marel, Phys. Rev. Lett. 100, 117401 (2008).

${ }^{11}$ Z. Q. Li, E. A. Henriksen, Z. Jiang, Z. Hao, M. C. Martin, P. Kim, H. L. Stormer, and D. N. Basov, Nat. Phys. 4, 532 (2008).

${ }^{12}$ R. R. Nair, P. Blake, A. N. Grigorenko, K. S. Novoselov, T. J. Booth, T. Stauber, N. M. R. Peres, and A. K. Geim, Science 320, 1308 (2008).

${ }^{13}$ H. Choi, F. Borondics, D. A. Siegel, S. Y. Zhou, M. C. Martin, A. Lanzara, and R. A. Kaindl, Appl. Phys. Lett. 94, 172102 (2009).
${ }^{14}$ F. Wang, Y. Zhang, C. Tian, C. Giri, A. Zettl, M. Crommie, and Y. Ron Shen, Science 320, 206 (2008).

${ }^{15}$ F. T. Vasko and V. Ryzhii, Phys. Rev. B 77, 195433 (2008).

${ }^{16}$ L. A. Falkovsky and S. S. Pershoguba, Phys. Rev. B 76, 153410 (2007); L. A. Falkovsky and A. A. Varlamov, Eur. Phys. J. B 56, 281 (2007).

${ }^{17}$ T. Stauber, N. M. R. Peres, and A. K. Geim, Phys. Rev. B 78, 085432 (2008)

${ }^{18}$ T. Ando, J. Phys. Soc. Jpn. 76, 024712 (2007).

${ }^{19}$ E. McCann and V. I. Falko, Phys. Rev. Lett. 96, 086805 (2006).

${ }^{20}$ W. Xu, F. M. Peeters, and T. C. Lu, Phys. Rev. B 79, 073403 (2009).

${ }^{21}$ W.-K. Tse and S. Das Sarma, Phys. Rev. Lett. 99, 236802 (2007).

${ }^{22}$ See, e.g., W. Xu, F. M. Peeters, and J. T. Devreese, Phys. Rev. B 43, 14134 (1991).

${ }^{23}$ See, e.g., W. Xu, Phys. Rev. B 71, 245304 (2005).

${ }^{24}$ See, e.g., X. L. Lei, J. Appl. Phys. 84, 1396 (1998).

${ }^{25}$ H. M. Dong, W. Xu, Z. Zeng, T. C. Lu, and F. M. Peeters, Phys. Rev. B 77, 235402 (2008).

${ }^{26}$ X. F. Wei, W. Xu, and Z. Zeng, J. Phys.: Condens. Matter 19, 506209 (2007).

${ }^{27}$ D. Sun, Z.-K. Wu, C. Divin, X. Li, C. Berger, W. A. de Heer, P. N. First, and T. B. Norris, Phys. Rev. Lett. 101, 157402 (2008).

${ }^{28}$ W. Xu, F. M. Peeters, and J. T. Devreese, Phys. Rev. B 48, 1562 (1993).

${ }^{29}$ See, e.g., S. Sheng, Li, Semiconductor Physical Electronics, 2nd ed. (Springer-Verlag, Berlin, 2006).

${ }^{30}$ H. J. Krenner, E. C. Clark, T. Nakaoka, M. Bichler, C. Scheurer, G. Abstreiter, and J. J. Finley, Phys. Rev. Lett. 97, 076403 (2006). 